\title{
ASOCIACIÓN ENTRE OBESIDAD E INFECCIONES: UN ESTUDIO DE CORTE TRANSVERSAL
}

\author{
Toledo José, Cubillos Gabriel', Gómez Oscar \\ ${ }^{1}$ Médico Psiquiatra, Epidemiólogo General, Máster Propio en Ensayos Clínicos, Profesor Universidad El Bosque \\ y Universidad Militar Nueva Granada. \\ ${ }^{2}$ Médico Cirujano Esteticista, Director Científico Clínica Colombiana de Obesidad. \\ ${ }^{3}$ Médico Cirujano Plástico, Clínica Colombiana de Obesidad. \\ Correspondencia: toledojose@unbosque.edu.co \\ Recibido: Noviembre 7 de 2013 Aceptado: Marzo 6 de 2014
}

\begin{abstract}
Resumen
Antecedentes: Desde hace más de dos décadas se reportan observaciones que plantean que la obesidad se asocia a la presencia de infecciones. Sin embargo, los diferentes hallazgos han sido contradictorios y la dirección de la posible asociación no ha sido clarificada. En nuestro país no se encontraron trabajos al respecto que contribuyan a aclarar esta pregunta.
\end{abstract}

Métodos: se realizó una serie de casos retrospectivo ( $\mathrm{N}=4840$ con muestreo por conglomerados $\mathrm{n}=100$ ), evaluando Índice de Masa Corporal (IMC) e infección por Helicobacter pylori, Streptococcus $\beta$ Hemolítico, Infección Urinaria (IVU) y Vaginosis. Se obtuvieron estadísticos descriptivos y se realizaron cruces de variables para obtener OR.

Resultados: $85 \%$ sexo femenino, procedentes de la zona andina en un 77\% que consultan en su mayoría para lipólisis laser, IMC: 32 (DS 5,4), la relación entre infección y obesidad fue: IVU OR 1,4 (IC 1,02-3,62), (p:0,042), VAGINITIS: OR 1,4 (IC: 1,09-3,019), (p:0,028), Helicobacter pylori: Pearson 0,25 (p:0,064) y Streptocccus: 0,56 (p:0.046).

Conclusiones: La presencia de infección Urinaria y la vaginitis se asocian a la obesidad. Estos hallazgos confirman previos estudios, se discuten las implicaciones de los mismos.

Palabras clave: infección, peso corporal, cirugía estética.

\section{ASSOCIATION BETWEEN OBESITY AND INFECTION: A STUDY OF CROSS SECTION}

\begin{abstract}
Background: From almost two decades ago, some reports had suggested that obesity is associated with active infections, however, these findings have been contradictory and the address of possible associations has not been clarified. In our country, no search was founded to clarify the question.

Methods: In a retrospective case series fashion ( $N=4840$ with conglomerate sampling $n=100$ ) we evaluate Body Mass Index (BMI) and infection by Helicobacter pylori , $\beta$ Hemolytic Streptococcus , Urinary Tract Infection (UTI) and Vaginosis. We get descriptive statistics and Odds Ratios.
\end{abstract}


Results: $85 \%$ female, 77\% from the Andean region $77 \%$,consulting mostly for laser lipolysis, BMI: 32 (SD.5.4), the relationship between infection and obesity was : UTI OR: 14 (CI 1.02 to 3.62 ) (p : 0.042 , vaginitis : OR 1.4 ( IC: $1,09-3,019)$, ( $:$ : 0.028), Helicobacter pylori: Pearson $0.25(p: 0.064)$ Streptococcus $0.56(p=0.046)$ These findings confirm previous studies. Implications are discussed.

Conclusions: in our search Urinary tract infections and vaginitis are associated with obesity, this data confirm previous reports.

Keywords: infection, body weight, esthetic surgery

\title{
ASSOCIAÇÃO ENTRE OBESIDADE E INFECÇÕES: UM ESTUDO TRANSVERSAL
}

\begin{abstract}
Resumo
Justificativa: Observações durante duas décadas sugerem uma relação entre obesidade e a presença de infecções, no entanto, os diferentes achados têm sido contraditórios e a associação ainda não foi verificada. No nosso país, não foram achadas pesquisas no tema.
\end{abstract}

Métodos: Numa série de casos retrospectiva ( $\mathrm{N}=4840$ amostragem por conglomerados com $\mathrm{n}=100$ ), avaliando do índice de massa corporal (IMC) e infecção pelo H. pylori.

Métodos: uma série de casos retrospectiva $(\mathrm{N}=4840$ amostragem por conglomerados com $\mathrm{n}$ $=100$ ), a avaliação do índice de massa corporal (IMC) e infecção pelo Helicobacter Pylori, Estreptococcus $\beta$ Hemolítico, infecção do trato urinário (ITU) e vaginose. As estatísticas descritivas foram obtidas e variáveis transversais foram realizadas para o odds ratio (OR).

Resultados: $85 \%$ do sexo feminino, $77 \%$ da região andina consultantes principalmente de lipólise por laser, IMC: 32 (DE 5.4), a relação entre infecção e obesidade foi: IVU OR 1,4 (IC 1,02-3,62) (p: 0,042), VAGINITE: OU 1,4 (IC: 1,09-3,019) (p: 0,028), H. pylori: Pearson de 0,25 (p=0,064) e Estreptococos 0,56 (p: 0,046).

Conclusões: A presença de infecção urinária e vaginite estão associadas com a obesidade. Estas constatações confirmam estudos anteriores, as suas conseqüências são discutidas.

Palavras-chave: Infecção, peso corporal, cirurgia estética.

\section{Introducción}

El progreso mundial acompañado de cambios en los patrones alimenticios se asocia a una situación de salud sin antecedentes: la obesidad. Esto ha llevado a las ciencias de la salud a replantear conceptos sobre las funciones y actividades de las células grasas que pasaron de ser conceptualizadas como almacenadoras de energía para además, producir moléculas asociadas a la inflamación crónica sistémica(1) que facilitan estados inflamatorios crónicos y por este mecanismo, colaborar en la aparición de variadas patologías. Existe evidencia obtenida por medio de diversos diseños que asocia la obesidad a la presencia de Herpes Simple (HSV) $(1,2)$ y otras enfermedades sugiriendo que la obesidad debería ser considerada al plantear estrategias y medidas de Salud Pública contra estas patologías.

Se investiga entonces, la asociación entre Helicobacter pylori y obesidad: para Furuta (3) la erradicación de $H$. pylori mejoraría parámetros nutricionales; se ha estudiado además, en su mayoría a través de series de casos, casos y controles y revisiones sistemáticas $(4,5,6)$ la asociación entre la dupla ghrelina - leptina y Helicobacter pylori con hallazgos contradictorios a la fecha que parecen indi- 
car que la erradicación de esta bacteria aumenta el peso corporal y la grasa libre (7). Casi todos estos trabajos han sido realizados a partir de observar que los pacientes ulcerosos que reciben tratamiento para Helicobacter pylori aumentan su masa corporal. Parece ser que las células grasas tienen un papel claro en la patogenia de procesos infecciosos, lo que abre un campo interesante de investigación en la relación entre la obesidad, el sobrepeso y los procesos infecciosos.

Profundizando en este campo, se han realizado investigaciones que pretenden evaluar infecciones en mujeres con hallazgos también contradictorios. En un análisis secundario, Masttrobatista (8) no encontró relación entre la persistencia de infección vaginal en mujeres embarazadas y el Índice de Masa Corporal (IMC). En varones, Saliba (9) en un estudio de cohortes encontró asociación entre obesidad y riesgo de infección urinaria.

Los estudios muestran que existe asociación entre obesidad e infección en humanos, pero encontrar una relación causal no ha sido conclusivo. Por un lado los individuos obesos presentan respuestas alteradas a la infección pero por otro lado algunos organismos patógenos parecen facilitar obesidad (HV, Helicobacter pylor, vaginosis etc). Para colaborar en esta búsqueda que tiene importantes efectos sobre la Salud Pública, se realizó un estudio descriptivo en una gran muestra de sujetos intervenidos en una la Clínica Colombiana de Obesidad.

En esta institución el tratamiento de la Obesidad se concibe como un proceso en el cual el acto quirúrgico y las actividades pre y post quirúrgicas son parte importante pero no única de un proceso que incluye: evaluación endocrina, psicológica, nutricional entre otros y tratamientos en caso de detectarse problemas en estos aspectos. Las evaluaciones pre y post quirúrgicas se realizan en un período de aproximadamente tres meses.

\section{Material y metodos}

Con un diseño descriptivo de serie de casos se tomó como universo la información existente en una base de datos que contiene las historias clínicas de todos los sujetos atendidos en la Clínica Colombiana de Obesidad entre 2008 y mayo de 2013 correspondiente a un total de 4840 sujetos. Se realizó un muestreo por conglomerados con el paquete estadístico EPI-INFO versión 6.1, asumiendo como conglomerado cada año (2010: 1349 sujetos, 2011: 974 sujetos, 2012: 1524 y 2013: 993) con una frecuencia esperada de $50 \%$ y un máximo de alejamiento aceptable de 10\% hacia arriba o hacia abajo y con una confianza de $99 \%$ se obtuvieron 100 sujetos (para 2010: 28, 2011: 20, 2012:32 y 2013:20). Los datos fueron obtenidos con un listado al azar por medio del paquete estadístico SPSS (versión 18), eligieron sujetos de reemplazo en caso de no tener datos insuficientes. Los datos fueron depurados independientemente por los autores y contrastados contra las historias clínicas físicas correspondientes. Los datos estadísticos se obtuvieron por medio del paquete SPSS versión 18 estimando los parámetros por medio de prueba de hipótesis e intervalos de confianza. Previa evaluación de la distribución, se realizaron pruebas de hipótesis adecuadas para evaluar los cambios obtenidos en IMC, MG y MM.

Para explorar la posible relación entre obesidad e infecciones se recodificaron las variables (IMC: normal y sobrepeso-obesidad) y las variables numéricas de acuerdo al punto de corte de presencia de infección aportado por el fabricante) y se construyeron tablas de $2 \times 2$ para obtener los respectivos $\mathrm{OR}$, se obtuvieron pruebas de hipótesis. Por tratarse de una investigación sin riesgo (estudio documental) no requirió consentimiento informado, sin embargo, en todo momento se mantuvo el anonimato de los sujetos de investigación.

Se evaluó la presencia de infecciones con las siguientes pruebas:

- Streptococcus determinación cuantitativa de antiestreptolisina, turbidimetría latex marca Spinreact ${ }^{\circledR}$ con especificidad $98 \%$ y sensibilidad $97 \%$.

- Helicobacter pylori: prueba rápida semicuantitativa, Estándard Diagnostic ${ }^{\circledR}$ sensibilidad 95\%, especificidad $85 \%$.

- Los diagnósticos de infección urinaria se hicieron con tira reactiva para uroanálisis Mission ${ }^{\circledR}$ y el diagnóstico de vaginosis se realizó por observación directa de frotis de flujo vaginal. Todos los exámenes fueron realizados por la misma bacterióloga.

\section{Resultados}

La información de los 100 sujetos fue obtenida de la base de datos disponible, la tabla 1 presenta las características demográficas con las frecuencias de proporción más altas de las variables categóricas. El paciente más frecuente es mujer, con pareja estable, procedente de la zona cundi - boyacense (zona geográfica en la cual está ubicada la Clínica Colombiana de Obesidad). En términos de tipo de ocupación, con trabajo de tipo estable. Por profesiones 
Tabla 1. Descripción de la población por variables categóricas

\begin{tabular}{|l|l|c|}
\hline \multicolumn{2}{|c|}{ Variables categóricas } & Proporción \\
\hline Sexo & Femenino & 85 \\
\hline \multirow{4}{*}{ Estado Civil } & Casado & 44 \\
\cline { 2 - 3 } & Union Libre & 42 \\
\hline \multirow{5}{*}{ Procedencia } & Bogota Y Altiplano & 77 \\
\cline { 2 - 3 } & Orinoquia & 5 \\
\cline { 2 - 3 } & Gran Antioquia & 4 \\
\cline { 2 - 3 } & Oriente & 2 \\
\hline \multirow{5}{*}{ Ocupacion } & Empleado & 30 \\
\cline { 2 - 3 } & Independiente & 13 \\
\cline { 2 - 3 } & Hogar & 9 \\
\cline { 2 - 3 } & Ingeniero & 9 \\
\cline { 2 - 3 } & Estudiante & 8 \\
\cline { 2 - 3 } & Comerciante & 8 \\
\cline { 2 - 3 } & Administrador & 7 \\
\cline { 2 - 3 } & Prof De Salud & 3 \\
\hline
\end{tabular}

Fuente: Base de datos llama la atención las frecuencias altas de Ingenieros, Administradores de Empresas y Profesionales de la Salud. Como este tipo de actividad quirúrgica no se encuentra contemplada en la Ley de Seguridad Social Colombiana no se presentan datos de tipo de aseguramiento.

En cuanto a las características cuantitativas más frecuentes de esta población, el paciente más frecuente tiene 36 años (IC: 34,4 - 37,8), mide $1,58 \mathrm{ctm}$ (IC 13) y pesa $70.4 \mathrm{kgr}$ (IC 11), con un IMC promedio de 32 (IC 5). En lo que respecta a los motivos de consulta, se exponen en la figura 1 , el ítem otros corresponde a celulitis, secuelas de lipoinyección y manchas en cara, como se aprecia 6 a 7 de cada 10 buscan cirugía para manejo de obesidad

La tabla 2 presenta los hallazgos más frecuentes relacionados con antecedentes patológicos y quirúrgicos de la muestra: del total de antecedentes quirúrgicos el $66,6 \%$ corresponden a antecedentes de cirugía plástica (liposucción, mamoplastia, lipectomía y lipólisis láser representan el 52\%).

Llama la atención también el porcentaje de patología tiroidea reportada. Los antecedentes Ginecológicos más frecuentes (eliminando de la base de datos los sujetos masculinos) se

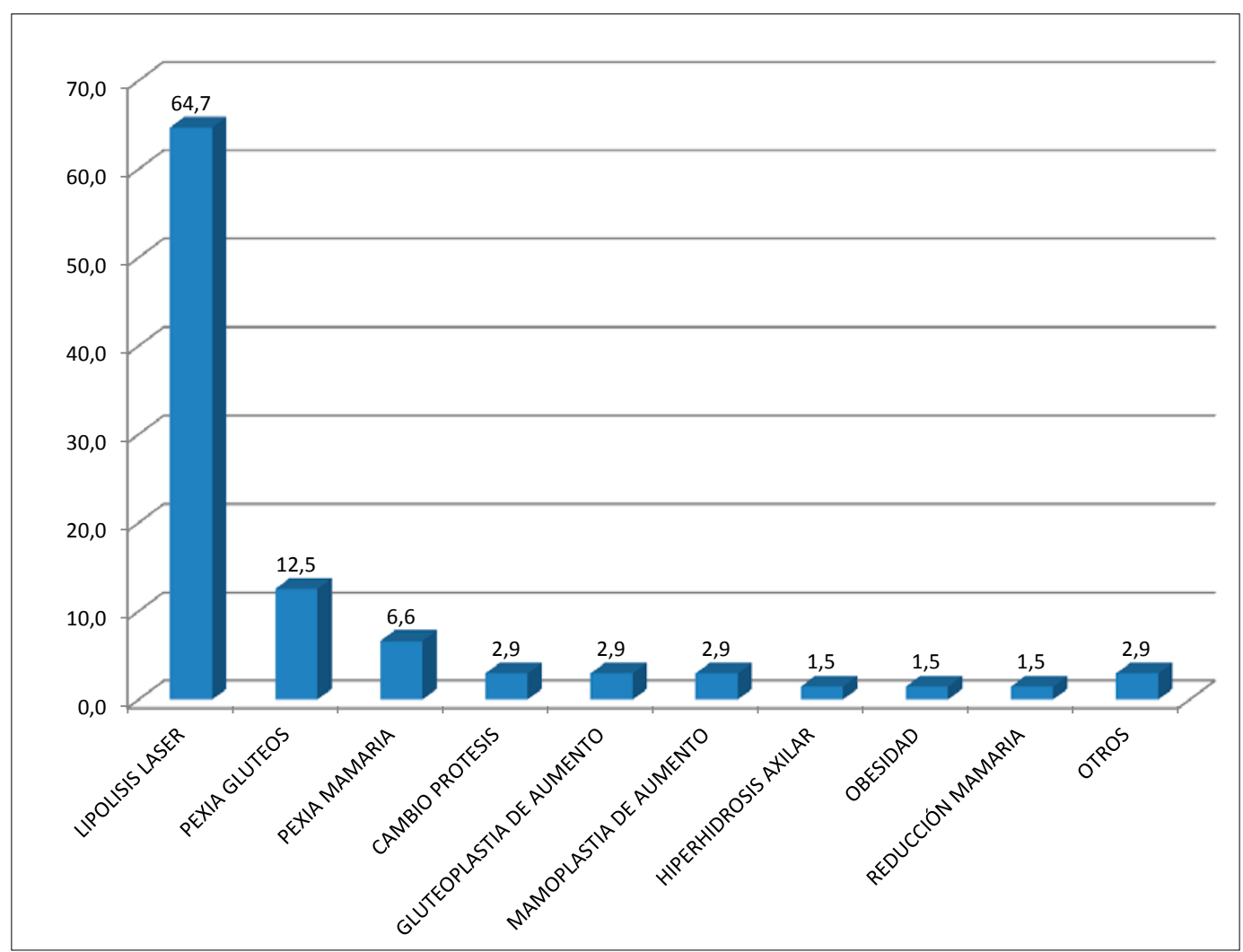

Figura 1. Motivos de consulta. Fuente: Base de datos 
Tabla 2. Antecedentes patológicos y quirúrgicos

\begin{tabular}{|l|c|l|r|}
\hline \multicolumn{1}{|c|}{ Patológicos } & $\mathbf{\%}$ & \multicolumn{1}{c|}{ Quirúrgicos } & \% \\
\hline Ninguno & 63 & Ninguno & 54 \\
\hline Tiroides & 7 & Cesarea & 53,5 \\
\hline Asma & 2 & Liposucción & 11,9 \\
\hline Hipertension & 2 & Mamoplastia & 11,5 \\
\hline Vph & 1 & Lipectomia & 8 \\
\hline Cardiopatia & 1 & Lipolisis Laser & 4 \\
\hline Migraña & 1 & Lifting Facial & 1,3 \\
\hline Diabetes gestacional & 1 & Colecistectomia laparoscopica & 1,3 \\
\hline
\end{tabular}

Fuente: Base de datos

Tabla 3. Antecedentes gineco-obstétricos

\begin{tabular}{|c|c|c|c|c|c|c|c|c|c|c|c|}
\hline 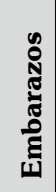 & $\%$ & 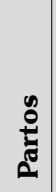 & $\%$ & 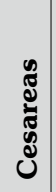 & $\%$ & 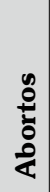 & $\%$ & $\begin{array}{l}0 \\
\frac{0}{5} \\
0 \\
0 \\
\frac{0}{0} \\
\frac{\pi}{z} \\
z\end{array}$ & $\%$ & 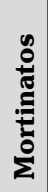 & $\%$ \\
\hline 0 & 41 & 0 & 62 & 0 & 59 & 0 & 71 & 0 & 38 & 0 & 83 \\
\hline 1 & 17 & 1 & 12 & 1 & 12 & 1 & 9 & 1 & 16 & 1 & 2 \\
\hline 2 & 25 & 2 & 19 & 2 & 13 & 2 & 5 & 2 & 27 & 2 & 1 \\
\hline 3 & 10 & 3 & 4 & 3 & 2 & 3 & 1 & 3 & 4 & 3 & 0 \\
\hline 4 & 5 & 4 & 2 & 4 & 0 & 4 & 0 & 4 & 1 & 4 & 0 \\
\hline 5 & 0 & 5 & 0 & 5 & 0 & 5 & 0 & 5 & 0 & 5 & 0 \\
\hline 6 & 1 & 6 & 0 & 6 & 0 & 6 & 0 & 6 & 0 & 6 & 0 \\
\hline ND & 0 & & 0 & & 13 & & 13 & & 13 & & 13 \\
\hline
\end{tabular}

Fuente: Base de datos

presentan en la tabla 3, además en términos de planificación el $43 \%$ no lo hace, $16 \%$ anticoncepción hormonal, $12 \%$ métodos de barrera incluyendo condón, $4 \%$ ligadura de trompas, 3\% DIU y el restante no entregó información.

En el uso previo de fármacos el 7\% aceptó usar vitaminas, $5 \%$ levotiroxina y el $4 \%$ algún tipo de tratamiento anti acné. Los hallazgos en examen físico más importantes se dieron en senos (Ptosis grado I 21\%, grado II 5\%, grado III $7 \%$, atrofia mamaria $6 \%$, prótesis 3 ), en abdomen (lipodistrofia II y III: $83 \%$ ) y en extremidades (Ptosis glútea Grado I: 26\% y Grado III: 5\%). La impresión diagnóstica se relaciona claramente con los hallazgos clínicos.

La tabla 4 presenta los cambios entre la etapa pre y post quirúrgica del tratamiento (de quienes recibieron trata-
Tabla 4. Cambios pre y postqururgicos

\begin{tabular}{|l|r|r|c|c|}
\hline \multicolumn{1}{|c|}{ Valor } & Imc & $\begin{array}{c}\text { Grasa } \\
(\mathbf{\%})\end{array}$ & $\begin{array}{c}\text { Masa } \\
\text { grasa }\end{array}$ & $\begin{array}{c}\text { Masa } \\
\text { magra }\end{array}$ \\
\hline Prequirurgico & 35,79 & 28,41 & 36,08 & 61,9 \\
\hline Postquirurgico & 24,44 & 25,45 & 15,85 & 44,88 \\
\hline \% de cambio & 31,72 & 11,42 & 47.44 & 28.15 \\
\hline P * & 0 & 0 & 0,002 & 0,345 \\
\hline
\end{tabular}

* Prueba de McNemar. Fuente: Base de datos

miento quirúrgico) tanto en el Indice de masa Corporal (IMC), el porcentaje de Grasa Total (GT), el porcentaje de Masa Grasa (MG) y el porcentaje de Masa Magra (MM). Todos estos cambios son estadísticamente significativos como puede observarse.

La tabla 5 presenta las proporciones de sujetos que tenían infecciones ya sea por Streptococcus $\beta$ Hemolytico, por Helicobacter pylori, infecciones Urinarias (IVU) o Vaginitis.

La tabla 6 presenta los Odds Ratio obtenidos para relacionar la presencia de Sobrepeso y Obesidad iniciales y la existencia de infecciones vaginal (eliminado los sujetos de sexo masculino) o urinaria (contando todos los sujetos). Además se recategorizó la variable en presencia de Obesidad únicamente, se aprecia como cambia el riesgo en función del aumento de peso:

Tabla 5. Pacientes con infecciones (\%)

\begin{tabular}{|l|c|c|}
\hline \multicolumn{1}{|c|}{ Infeccion } & Positivo & Negativo \\
\hline Estreptococo b hemolitico & 20 & 80 \\
\hline Helicobacter pylori & 37 & 63 \\
\hline Parcial de orina & 8 & 92 \\
\hline Vaginitis & 45 & 55 \\
\hline
\end{tabular}

Fuente: Base de datos

Tabla 6. Sobrepeso u obesidad e ivu o vaginitis

\begin{tabular}{|l|l|c|c|}
\hline $\begin{array}{c}\text { Factor de } \\
\text { riesgo }\end{array}$ & Infección & Odds Ratio (I.C.) & $\begin{array}{c}\text { P }(\mathbf{C H I} \\
\text { cuadrado) }\end{array}$ \\
\hline \multirow{2}{*}{$\begin{array}{l}\text { Sobrepeso u } \\
\text { obesidad }\end{array}$} & $\begin{array}{l}\text { Infección de } \\
\text { vias urinarias }\end{array}$ & $2,1(0,62-9,25)$ & 0,272 \\
\cline { 2 - 4 } & Vaginitis & $0,54(0,208-1,44)$ & 0,162 \\
\hline \multirow{2}{*}{ Obesidad } & $\begin{array}{l}\text { Infeccion de } \\
\text { vias urinarias }\end{array}$ & $1,4(1,02-3,62)$ & 0,042 \\
\cline { 2 - 4 } & Vaginitis & $1,4(1,09-3,019)$ & 0,028 \\
\hline
\end{tabular}

Fuente: Base de datos 
La tabla 7 ofrece los coeficientes de correlación de Pearson obtenidos entre el IMC y las pruebas de laboratorio usadas para detectar infección por Streptococus o por H. pylori.

Tabla 7. Asociacion entre sobrepeso - obesidad inicial e infeccion

\begin{tabular}{|l|c|c|c|}
\hline \multicolumn{1}{|c|}{ Organismo } & $\begin{array}{c}\text { Correla- } \\
\text { ción } \\
\text { de Pearson }\end{array}$ & $\begin{array}{c}\text { Coeficien- } \\
\text { te de } \\
\text { Regresión }\end{array}$ & p \\
\hline Helicobacter pylori & 0,25 & 0,0625 & 0,064 \\
\hline Streptocccus & 0,56 & 0,313 & 0.046 \\
\hline
\end{tabular}

Fuente: Base de datos

Como se aprecia, existe asociación entre la presencia de sobrepeso $\mathrm{u}$ obesidad y la presencia de infecciones.

\section{Discusión}

El presente estudio de serie de casos fue realizado en una muestra con $99 \%$ de confianza, dada la calidad de la información recolectada no fue necesario usar el listado de reemplazo de sujetos. El paciente típico es mujer, procedente del altiplano cundiboyacense en la cuarta década de la vida, con unión de pareja estable con trabajo estable. Si es profesional cumple labores que le implican contactos frecuentes con la gente, con hijos y con un alto porcentaje de cesáreas previas. Tiene estatura promedio para la población colombiana e IMC en Obesidad y que consulta básicamente buscando ayuda por este motivo. Llama la atención la proporción de hipotiroidismo encontrada (sin evaluar hipotiroidismo subclínico, congénito o no) ya que la reportada en Colombia varía entre 1/2250 a 1/9000 en hipotiroidismo congénito y $18.5 \%$ y la mundial hasta $1.8 \%(9,10)$.

Aunque los métodos de diagnóstico son diferentes en los reportes lo que hace difícil la comparación sus antecedentes quirúrgicos (excepto cesárea) están relacionados con sobrepeso. Estos hallazgos justifican plenamente el hecho de que el manejo de la obesidad no sea una actividad quirúrgica únicamente; debe incluir un plan global de tratamiento con cambios importantes en hábitos de vida. Se requiere investigación en este campo para definirlos y poderlos realizar intervención

Como indican los resultados de nuestro trabajo, el tratamiento integral permite un cambio importante en el IMC medido a los tres meses de realizado el acto quirúrgico. Como es de esperar el cambio más importante se hace a expensas de la masa grasa.
Los datos sobre prevalencia de infección de Vías Urinarias encontrada fue de $8 \%$, vaginitis $45 \%$ e infecciones por Streptococcus $20 \%$ y H. pylori de $37 \%$.

La comparación de estos datos con los obtenidos en otras publicaciones es difícil ya que varían los métodos diagnósticos así como los objetivos y los diseños publicados. En libros aceptados como centrales en los temas $(11,12)$, se encuentran los siguientes estadísticos de prevalencia: Bacteriuria: $1-3 \%$, Vaginitis $60-70 \%$, Estreptococcus $10-40 \%$ y de $H$. pylori $60 \%$ para personas en el mismo rango de edad, la comparación sin embargo es difícil por las razones anotadas. Los autores no encontraron datos similares en Colombia.

Este estudio falló en encontrar asociación entre sobrepeso e infección, pero muestra claramente como existe una asociación clara entre obesidad e IVU y vaginitis planteando que el aumento de peso está claramente relacionado con IVU y vaginitis, particularmente cuando la persona se encuentra en situación de obesidad.

En cuanto a asociación entre la presencia de H. pylori y sobrepeso $u$ obesidad el estudio de Ming-Shiang et al(13) no encontró asociación entre obesidad (mórbida para este estudio) y $H$. pylori pero encuentran como en este estudio baja seroprevalencia, planteando la hipótesis de que la pérdida de seropositividad en la infancia pudiese aumentar el riesgo de desarrollo de obesidad mórbida. (Estudio de casos y controles).

Creemos que se requiere mayor investigación en el tema probablemente estratificando grupos de obesidad y evaluando posibles tratamientos previos para H. pylori como factor de confusión y evaluando el efecto de la ghrelina sobre la leptina.

La asociación entre Streptococcus y obesidad es fuerte. Este hallazgo difiere del encontrado por Mastrobattista J. et al (9) explicable por la diferencia de diseños (el nuestro es una serie de casos y el referenciado es un Clinical Trial cuyo objetivo fue evaluar el efecto del IMC sobre el tratamiento con metronidazol de vaginitis en mujeres embarazadas). Sin embargo es claro en ambos estudios que el IMC aumentado con sus efectos sobre la distribución de fármacos y consecuente biodisponibilidad de los mismos, actúa directamente sobre las posibles patologías presentes en estos sujetos.

Aunque los datos del presente estudio encuentran asociación entre infección por Streptococcus, IVU y vaginitis, fallando en encontrarla en $H$. pylori, esta asociación por el tipo de diseño no puede ser interpretada como causal. 
Sin embargo implica que los médicos tratantes de estas pacientes pongan especial cuidado en buscar patologías de este tipo y tratarlas adecuadamente dentro de un proceso de tratamiento racional ya que como lo plantean Desruisseaux $S$ et al (15) el tejido adiposo y los adipocitos contribuyen significativamente a la interacción entre el organismo y agentes infecciosos, por la producción de sustancias que contribuyen directamente con los procesos infecciosos. Es claro que el tema requiere mayor investigación.

\section{Referencias}

1. Ramalho R, Guimaraes C. The role of adipose tissue and macrophages in chronic inflammation associated with obesity: clinical implications. . Acta Med Port. 2008; 21(5): 489 - 96. PMID: 19187692

2. Karjala Z, Neal D, Rohrer J (2011) Association between HSV1 Seropositivity and Obesity: Data from the National Health and Nutritional Examination Survey, 2007-2008. PLoS ONE 6(5): e19092.

3. Fernández-Real J, Ferri MJ, Vendrel J, Ricart W. Burden of infection and fat mass in healthy middle-aged men. Obesity (Silver spring) 2007, Jan. 15 (1): 245 - 52).

4. Furuta T, Shirai N, Xiao F, Takashima M, Hanai H. Effect of Helicobacter Pyliori infection and its eradication on nutrition. Aliment Pharmacol Ther 2002 Apr; 16(4):799-806.

5. Tan HJ, Goh KL. Extra gastrointestinal manifestations of Helicobacter pylori infection: Facts or myth? A critical review. J. Dig. Dis. 2012 Jul; 13 (7), 342-9.
6. Blanco Quiroz A., Obesidad y respuesta inflamatoria. Bol Pediatr 2007; 47: 237-49

7. Wu S, Lee WJ, Wang HH, Huang SP, Lin JT. A case-control study of association of Helicobacter pylori infection with morbid obesity in Taiwan. Arch Intern Med. 2005 Jul 11;165 (13):1552-5.

8. Kebapcilar L, Sari I, Renkal AH, Alacacioglu A, Yuksel A, Ilhan E, et al. The influence of Helicobacter pylori eradication on leptin, soluble CD40 ligand, oxidative stress and body composition in patients with peptic ulcer disease. Intern Med. 2009;48(24):2055-9. PMID: 20009392.

9. Mastrobattista JM, Klebanoff MA, Carey JC, Hauth JC, Macpherson CA, Ernest J, et al. The effect of body mass index on therapeutic response to bacterial vaginosis in pregnancy. Am J Perinatol. 2008 Apr;25(4):233-7.

10. Saliba W, Barnett-Griness O, Rennert G. The association between obesity and urinary tract infection. Eur J Intern Med. 2013 Mar;24(2):127-31.

11. Asociación Colombiana de Endocrinología. Consenso Colombiano para el diagnóstico y manejo de enfermedades tiroideas. Act Méd Col. Vol $24 \mathrm{~N}$ 4. Jul-Ago 1999

12. Londoño A. Prevalencia de hipotiroidismo y relación con niveles elevados de anticuerpos antiperoxidasa y yoduria en poblaciones de 35 y más años en Aremenia 2009, 2010. Rev Sal Púb. Universidad Nacional de Colombia. Vol 13 N 6.2011 pp. 998 - 1009

13. Mendell G. Principles and practice of infectious diseases. 4th ed. Baltimore. Springler. 2010.

14. Decharny. Current diagnosis and treatment of gynecologic diseases. New York. McGraw Hill.2013.

15. Desruisseaux MS, Tanowitz HB, Scherer PE. Adipocyte, adipose tissue, and infectious disease. Infect Immun. 2007 Mar;75(3):1066-78. 